\title{
Germanica
}

\section{La Petite fille aux allumettesde Helmut Lachenmann. Un « spectacle de la perception »}

Das Mädchen mit den Schwefelhölzern von Helmut Lachenmann, ein «Wahrnehmungsstück».

Joëlle Caullier

\section{OpenEdition}

\section{Journals}

Édition électronique

URL : http://journals.openedition.org/germanica/479

DOI : $10.4000 /$ germanica. 479

ISSN : 2107-0784

\section{Éditeur}

Université de Lille

\section{Édition imprimée}

Date de publication : 1 décembre 2007

Pagination : 131-149

ISBN : 978-2-913857-20-9

ISSN : 0984-2632

Référence électronique

Joëlle Caullier, «La Petite fille aux allumettesde Helmut Lachenmann. Un « spectacle de la perception » », Germanica [En ligne], 41 | 2007, mis en ligne le 01 décembre 2009, consulté le 06 octobre 2020. URL : http://journals.openedition.org/germanica/479 ; DOI : https://doi.org/10.4000/ germanica.479

Ce document a été généré automatiquement le 6 octobre 2020.

(c) Tous droits réservés 


\title{
La Petite fille aux allumettesde Helmut Lachenmann. Un « spectacle de la perception »
}

\author{
Das Mädchen mit den Schwefelhölzern von Helmut Lachenmann, ein \\ «Wahrnehmungsstück».
}

Joëlle Caullier

J'ai profité de l'opportunité d'un sujet archétypal,
connu, et en même temps riche d'actualité, qui
me permettait de mettre radicalement au centre
d'un « spectacle de la perception » une musique
radicale. L'opéra du futur ne pourra pas consister
en un arrangement subtil d'affects standardisés,
quel que soit leur contexte. Je ne pourrais jamais
composer un opéra à contenu dramatique ${ }^{1}$.

Es war fürchterlich kalt... Dès les premiers mots du conte, une sensation douloureuse de froid glacial saisit le lecteur d'Andersen comme elle assaille l'auditeur de l'œuvre de Helmut Lachenmann ${ }^{2}$. Dès les premières minutes, le spectateur se trouve transi par une musique l'obligeant à se recroqueviller dans une encoignure de lui-même, comme par empathie avec le petit être misérable et solitaire qui erre lamentablement dans cette nuit d'hiver avant de se laisser mourir contre un pan de mur gelé... Quatre petites pages de conte destinées à l'intimité d'une lecture familiale édifiante vont suffire à inspirer, dans le lieu le plus symboliquement socialisé qu'est l'opéra, l'une des œuvres les plus arides et les plus exploratoires que l'art contemporain ait vu naître.

2 Il est vrai qu'élire un conte d'Andersen pour argument d'un opéra ne constitue pas, à l'extrême fin $\mathrm{du} \mathrm{xx}^{\mathrm{e}}$ siècle ${ }^{3}$, un acte banal, surtout lorsqu'on choisit d'y apposer le langage musical le plus radical qui soit! Y «apposer »... Est-ce d'ailleurs bien là le terme qui convient à la démarche d'un compositeur qui s'est toujours défié des mises en voix de textes, défié de toute voix, tout simplement, comme de tout contenu dramatique? Ne devrait-on pas lui préférer d'autres termes, plus violents mais sans 
doute plus justes : " en extraire ", « en arracher ", « en extirper »..., tant le musicien traverse le texte, le déchire, le troue, pour y chercher sans concession la trace sensorielle de la morsure du froid et de l'abandon, et l'imprimer par la force des sons dans le corps même de l'auditeur?

Comment un compositeur aussi vigilant que Lachenmann aux possibles récupérations de l'art le plus contestataire par la consommation culturelle a-t-il finalement pu se résoudre à composer pour l'Opéra, qui plus est sur un texte familier, venu de l'un des conteurs romantiques européens les plus célébrés ? Car le livret est bien, en effet, le minuscule texte d'Andersen, aussi minuscule que la petite fille qui exhale seule son dernier souffle, dans l'indifférence du monde, une nuit de la Saint-Sylvestre. Le récit nous en sera livré intégralement, quoique déconstruit linguistiquement par le compositeur, sans les démystifications que la littérature $\mathrm{du} \mathrm{xx}^{\mathrm{e}}$ siècle s'est plu à faire subir aux mythes et aux contes (songeons par exemple à la Schneewittchen de Robert Walser, récemment devenue opéra sous la plume de Heinz Holliger). Comment le conte d'Andersen, si touchant mais si pudique, a-t-il pu devenir l'une des œuvres les plus ardues et les plus dérangeantes de la modernité européenne, pourtant fort peu encline aux états d'âme et aux bons sentiments? Quelle démarche artistique inédite a-t-il su mobiliser et à quelles fins?

\section{L'œuvre}

4 Avant de méditer sur cette œuvre hors du commun et en particulier sur son livret, sans doute est-il nécessaire d'en proposer une brève présentation.

5 Tout d'abord, il faut y insister, La Petite Fille aux allumettes n'est pas un opéra à proprement parler ${ }^{4}$. Bien que l'Opéra de Hambourg ait passé commande au compositeur dès 1988, l'œuvre, qui voit enfin le jour en 1997 après des années de doute et maints découragements ${ }^{5}$, porte le sous-titre de « Musik mit Bildern» (« Musique avec images »). En refusant le genre opéra à proprement parler, Lachenmann manifeste sa crainte de voir son œuvre récupérée non seulement par l'industrie culturelle mais aussi par une certaine manière bourgeoise, confortable, de ramener toute nouvelle œuvre au déjà-connu, consommable sans danger, expurgée de son contenu dérangeant. À juste titre sans doute, l'opéra cristallise la méfiance du compositeur pour deux raisons essentielles: d'une part, il est le genre par excellence par lequel la bonne société se représente elle-même, nantie de ses valeurs; de l'autre, il consacre le règne de la voix. Tout ce qui fait appel à la voix chantée en musique est par nature, selon Lachenmann, citation et auto-glorification de l'homme. Or, la recherche du compositeur s'exerce, elle, dans l'intention de créer un univers nouveau ${ }^{6}$, critique, à proprement parler inouï, pour lequel la "désemphatisation" de l'instrument-voix et sa libération des conventions vocales sont absolument nécessaires; tout comme est nécessaire l'élaboration d'une écoute subtile et renouvelée du monde sonore, seule susceptible de réveiller la sensorialité contemporaine anesthésiée par l'omniprésence du bruit, la banalisation du fait musical et le conformisme auditif. Tout le travail du compositeur tend donc à la rééducation sensorielle de ses contemporains, non à des fins hédonistes, mais dans le but d'exhumer des facultés endormies, en dévoilant le caractère profondément incarné de la pensée lorsqu'elle se nourrit d'une expérience humaine pleine et entière. 
6 Dès la conception de l'œuvre, Lachenmann développe une analyse politique du sujet quoiqu'il se soit toujours montré réfractaire à l'art engagé comme reflet de la réalité sociale, tout en partageant la conscience critique des intellectuels de sa génération. Il faut dire que l'Allemagne vient de vivre intensément le procès du groupe BaaderMeinhof dont l'action terroriste avait secoué les années 70. Le compositeur, qui tient à ancrer son œuvre dans la vie commune, met en lumière une interprétation politique du conte d'Andersen, mais une interprétation censée renouveler les réactions des auditeurs plutôt que susciter une révolution sociale jugée illusoire. C'est ce qui explique la surprenante insertion de tropes, fragments textuels totalement étrangers au conte, dans le livret: d'une part, un écrit de révolte politique de Gudrun Ensslin, l'une des terroristes de la Fraction Armée rouge qui avait été sur les bancs de l'école avec Lachenmann et qui se suicidera en prison en 1977, d'autre part deux textes de Léonard de Vinci où l'artiste médite sur les mystères effrayants de la nature et les sentiments contradictoires qu'ils éveillent en lui (Zwei Gefühle ${ }^{7}$ ). Ces tropes se substituent partiellement aux hallucinations de la petite fille dans le conte d'Andersen.

7 Qu'on ne s'attende pas à une œuvre théâtrale traditionnelle, avec des personnages, une narration linéaire et des situations dramatiques! Il serait en effet erroné de dire que l'œuvre met en scène la petite fille. Au cœur d'un récit et non d'un drame scénique, celle-ci n'est pas pensée comme un personnage et, lors de la représentation, peut se trouver hors de vue du public. Aucun chanteur soliste ne l'incarne, mais deux voix juvéniles l'évoquent. Pourtant, sa présence est partout, par la trace de son ressenti et l'empathie qui s'empare du spectateur. Des voix collectives donnent parfois à entendre des bribes de mots qu'elle a dû prononcer et que le conteur ajoute pour vivifier sa version (Kauft!), mais aucune parole distincte ne lui est attribuée. On n'identifie pas non plus le timbre unique d'une voix de narrateur. Le véritable conteur est la musique ellemême, voix et instruments mêlés. Les voix présentes sont toujours collectives, fondues par leur caractère bruitiste à un univers instrumental complexe qui souligne ou prolonge leur structure phonétique (frottements des instruments pour souligner les chuintantes, roulements ou trémolos sur les $r$ roulés, assonances instrumentales diverses...) et si la partie vocale s'appuie scrupuleusement sur le texte d'Andersen, elle le déforme de telle façon, en disloquant les mots, les décomposant en phonèmes euxmêmes conglomérés - à l'encontre de toute perception sémantique ${ }^{8}-$, qu'il n'est pas question de le « comprendre "; l'auditeur doit faire des efforts démesurés pour suivre un texte qu'il connaît pourtant quasiment par cœur depuis sa petite enfance. Mais en même temps, cette difficulté de compréhension rationnelle fait surgir chez le spectateur des capacités insoupçonnées de compréhension d'un autre ordre, plus intuitif et affectif, celui-là :

«Ce que l'on va revivre », dit Lachenmann, «c'est le son, gorgé d'affects, qui s'en [le texte d'Andersen] inspire. La première réplique de mon opéra dit: "Il faisait un froid terrible". Cela est une simple information, mais relève aussi d'une sorte de liturgie du conte. Chez moi, elle est distendue phonétiquement et articulée de manière à ce qu'une perception vraiment aux aguets, qui ne fait pas "qu'écouter" de manière passive, puisse déchiffrer ce texte, à l'intérieur d'un moment très étiré. L'écoute comme déchiffrage : voilà un appel qui s'adresse à la perception autant qu'à la mémoire, et quand les deux sont à l'œuvre, on comprend davantage dans $L a$ Petite Fille que dans d'autres opéras. [...] Les paroles ont une parenté naturelle avec ce qu'elles expriment. Bien sûr la petite fille ne chante pas "j'ai froid", mais elle tremble, elle halète, elle cherche à respirer, et c'est toujours le son pur, le phonème brut qui représente le message. [...] Tout cela vaut aussi pour Gesualdo et même 
pour Bach. La "compréhension" purement sémantique du contenu ne garantit pas de toute façon qu'on l'ait compris. C'est l'interaction de l'image, de la lumière, du mouvement, de l'action, du son, de la structure musicale - c'est cette expérience sensible très complexe qui est le véhicule du sens ${ }^{9}$ ".

Le temps du récit est de ce fait complètement transformé par le temps musical qui s'étire lentement, à l'image de l'insidieuse souffrance imposée à l'enfant par cette interminable nuit glaciale. Pour Lachenmann comme dans les pratiques traditionnelles de contes où la plupart des auditeurs connaissent déjà l'histoire, " c'est une narration où les spectateurs n'écoutent plus, mais observent le narrateur en train de raconter $»^{10}$. Témoins de l'être au présent et non d'une action rapportée (c'est d'ailleurs cet intérêt pour l'être qui rend le découpage en actes inadéquat dans l'opéra contemporain), les tableaux-images auxquels fait allusion le titre de Musik mit Bildern reflètent non des situations réalistes, mais des moments flottants, non reliés par le flux de la conscience, captés dans l'imaginaire même de la petite fille. Et ce sont eux qui, sous forme d'images sonores, s'insinuent dans l'être du spectateur grâce à la médiation active du conteurorchestre. D'où le choix du compositeur de montrer les instrumentistes et chanteurs, ombres de l'imaginaire silencieux de l'enfant, plutôt que les personnages en tant que tels. D'où également son choix de distribuer une partie de l'effectif instrumental dans la salle, parmi les auditeurs, afin de créer le milieu propice - obscurité et bruissement environnant - à des synesthésies auditives, visuelles et tactiles supposées favoriser chez le spectateur le déploiement d'un imaginaire sensoriel particulièrement actif.

Dix tableaux se succèdent dans la première partie («Dans la rue ») consacrée à la rude journée passée dehors, dix tableaux saisissant la réalité d'états sensoriels vécus par la petite fille : elle entend des chants de Noël ( $\mathrm{d} u$ fröhliche, Stille Nacht...), souffre du froid (Air et trio du froid), claque des dents (Aria des claquements de langue), évite de justesse des voitures qui manquent de l'écraser (La Chasse), tente malgré son menton gelé et sa faim de héler de potentiels acheteurs d'allumettes (le Kauft devient une sorte de leitmotiv) et regarde par les fenêtres éclairées les préparatifs de la fête...

Quatorze tableaux constituent la deuxième partie («Au pied du mur de la maison ») où la petite fille se blottit contre un mur qui va se mettre à refléter les hallucinations produites par la flamme éphémère des allumettes qu'elle craque une à une, jusqu'à ce qu'elle-même s'éteigne... Mais à deux reprises, les hallucinations d'Andersen se voient remplacées par des textes inconnus qui semblent émerger - comme révélés par une encre sympathique - du mur gris et glacé, fugitivement animé par la flamme: la dénonciation d'une société elle-même criminelle et criminogène ${ }^{11}$ par Gudrun Ensslin, un discours comme pétrifié, automatisé, et - avant l'apparition de la grand-mère salvatrice - la méditation métaphysique de Léonard de Vinci ${ }^{12}$ fasciné par l'inquiétante étrangeté d'une caverne obscure qui éveille en lui deux sentiments contradictoires (zwei Gefühle), la peur de la mort et le désir platonicien de percer l'obscurité pour comprendre le mystère... Ces deux fragments "extra-territoriaux", comme dirait Adorno, illustrent dans le livret le procédé du montage ${ }^{13}$ cher aux expressionnistes qui cherchaient, par des césures brutales, à faire surgir de l'hétérogénéité des matériaux choisis un sens renouvelé. Le conteur laisse ainsi affleurer sa personnalité en insérant son commentaire propre sous cette forme distanciée du montage.

11 Musicalement ${ }^{14}$, l'œuvre mobilise 2 sopranos, 4 groupes de 8 voix mixtes constituées en quatuor et un immense orchestre composé de cordes, bois, cuivres, 5 percussionnistes, orgue électrique, célesta, 2 pianos, 2 guitares électriques et 2 harpes, un octuor à cordes soliste en plus des cordes de l'orchestre, 6 bandes pré-enregistrées (comprenant tant 
des extraits musicaux que des fragments parlés et des bruits blancs), un orgue à bouche japonais et de nombreux ustensiles que les instrumentistes sont amenés à manier (plaques de polystyrène, brosses, balais, marteaux, tissus...). Mais cet effectif imposant, outre qu'il est rarement utilisé à plein registre, enveloppe le spectateur en grignotant la salle. Comme dans le Prometeo de Nono (le maître et ami du compositeur), des îlots musicaux répartis dans l'espace total du théâtre immergent l'auditeur dans une mer de sons, détournant son attention d'une scène sur laquelle ne se passe pas l'essentiel et mobilisant comme jamais l'ensemble de ses facultés perceptives.

Lachenmann recourt ici, loin de tous les systèmes élaborés par les générations musicales successives (tonalité, modalité, atonalité libre ou polarisée, sérialisme, musiques spectrale, aléatoire, électracoustique...), au langage qui lui est propre, la musique concrète instrumentale. Elle éclaire d'un autre jour encore ce terme de Bilder revendiqué par le compositeur, images sonores évocatrices du ressenti individuel. Instruments et voix sont utilisés de manière non conventionnelle de façon à produire, avec des instruments traditionnels ou avec des accessoires divers, des sonorités inouïes qui ont pour fonction primordiale de renvoyer l'auditeur à son expérience corporelle propre : ainsi la traduction du froid est-elle réalisée par des frottements de cordes et de mains, des souffles, des répétitions frissonnantes de consonnes $(w)$, des harmoniques, des onomatopées imitant le vent..., le craquement de l'allumette ( $r i t s c h »)$ est métaphorisé par des percussions en bois, des pizzicati en harmoniques aux cordes, des frottements à l'intérieur du piano..., la poursuite en voiture rendue par des gammes et glissandi divers, notamment aux trombones, des coups de baguettes sur les pupitres imitent le fouet claquant sur les chevaux... De nombreuses citations musicales passent furtivement pour faire sens : l'air de la Reine de la nuit sur la consonne $f$ venue du mot kauft qu'essaie de prononcer la petite, transie, cherchant à vendre ses allumettes (Kauft Streichhölzer!), Stille Nacht chanté avec des claquements de langue... Des images sonores qui rappellent le figuralisme des " airs de froid» baroques, autant que les sensations d'engloutissement des voix d'enfants du Gesang der Jünglinge de Stockhausen ${ }^{15}$... Par ces imitations et métaphores auxquelles voix et instruments contribuent à part égale, Lachenmann cherche à rester au plus près de l'expérience sensorielle partagée, de façon à solliciter la mémoire corporelle de l'auditeur et à le toucher ainsi dans son for le plus intime. Il lui ouvre par ailleurs des pistes de réflexion en provoquant des questionnements stimulants : pourquoi, par exemple, la référence énigmatique à la Reine de la nuit? Est-ce de l'humour noir et une manière de dire que la petite marchande d'allumettes seule dans la rue est la reine de cette nuit-là ou est-ce un avertissement devant le fait que l'injustice intolérable risque d'amener la métamorphose de l'opprimée en furie vengeresse et destructrice - Gudrun Ensslin, en l'occurrence ? On le voit donc, de la mémoire sensorielle au projet politique, la musique de Lachenmann s'inscrit pleinement dans une analyse critique de la société et dessine l'utopie d'une transformation profonde de l'homme par le réveil de ses facultés perceptives, une transformation que ni l'humanisme, ni les révolutions, ni les combats idéologiques n'ont été capables d'accomplir. Car la visée est bien d'agir sur la perception pour construire une autre réalité.

\section{L'art de conter}

Un seul homme peut prétendre avoir quelque connaissance de la vie dans ce qui fait sa 


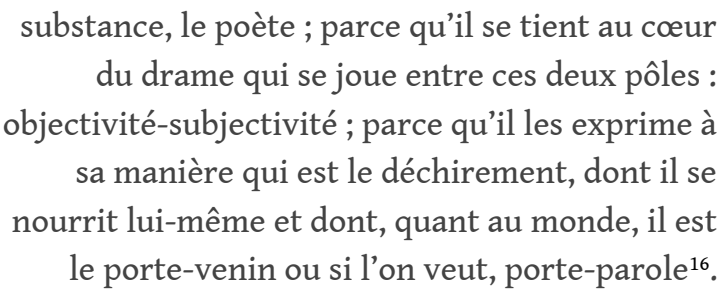

13 Le genre du conte ne semble pas faire bon ménage avec la scène d'opéra. Lorsqu'il y accède, c'est généralement privé de son caractère propre de récit et travesti sous une forme dramatique qui lui est précisément totalement étrangère. Ainsi La Cenerentola de Rossini, Hänsel und Gretel de Humperdinck, Turandot de Puccini, Pollicino de Hans Werner Henze... mettent-ils en scène des personnages qui jouent leur propre histoire mais qui ont éliminé leur conteur. Rares sont les œuvres musico-scéniques $\mathrm{du} \mathrm{xx}^{\mathrm{e}}$ siècle qui conservent du conte sa forme particulière de récit, fort peu propice, il faut bien le reconnaître, à la représentation théâtrale. Sans doute aussi, le $\mathrm{xx}^{\mathrm{e}}$ siècle ne croit-il plus au merveilleux qui n'a pu résister à la terrible lucidité imposée par Auschwitz et le cortège de catastrophes collectives de l'époque.

Mais les exigences de la scène ne sont certainement pas seules à expliquer la rareté du conte à l'Opéra. Dans son article fondamental, «Le Conteur. Réflexions sur l'œuvre de Nicolas Leskov", Walter Benjamin analyse magistralement comment le retrait de l'expérience propre aux temps modernes entraîne à sa suite une désaffection pour l'art de conter ${ }^{17}$. «L'expérience transmise de bouche en bouche est la source à laquelle tous les conteurs ont puisé.» Or, la société industrielle et ses techniques de production fondées sur l'atomisation des tâches et la division du travail, ont peu à peu privé les hommes de l'expérience de la vie dans sa totalité, cette carence se manifestant dans la quasi disparition de l'art de raconter qui lui-même supposait une expérience partagée et la conscience de faire partie d'une communauté humaine. L'avalanche moderne d'informations s'est ajoutée à cet état de fait, plongeant l'individu dans une immédiateté des événements qui rend obsolète la médiation d'un conteur, passeur d'expériences. Car "le récit ne vise point à transmettre le pur "en soi" de la chose, comme une information ou un rapport. Il plonge la chose dans la vie même du conteur et de cette vie ensuite la retire. Le conteur imprime sa marque au récit comme le potier laisse sur la coupe l'empreinte de ses mains ${ }^{18}$ ». Par ailleurs, "c'est de la mort que le conteur tire son autorité, car il transmet l'expérience ultime de ses personnages, celle qui ne peut être qu'essentielle puisqu'elle est révélée au moment suprême. En subordonnant le récit aux insondables desseins de la Providence divine, les chroniqueurs se sont d'emblée déchargés du soin de démontrer et d'expliquer. Ils y suppléent par l'interprétation, qui ne vise pas à enchaîner rigoureusement les événements les uns aux autres, mais à les insérer dans le cours insondable du monde ${ }^{19}$ ".

D'après ce qui a déjà été dit de l'œuvre de Lachenmann et ces méditations de Benjamin, le lecteur comprendra la pertinence du conte aux yeux du compositeur, lui qui déploie dans son activité musicale une réflexion pratique et qui cherche à réveiller chez ses contemporains une lucidité nourrie d'une expérience ravivée. Encore faut-il que cette expérience ne soit pas « virtualisée » par la fugacité d'informations multiples et qu'elle trouve à s'incorporer en chaque auditeur. D'où la préoccupation centrale de Lachenmann pour la réactivation d'une perception qui peu à peu avait été émoussée par l'habitude. 
16 Il choisit donc de faire redécouvrir à ses auditeurs un conte que chacun connaît mais qui hélas n'éveille toujours qu'une compassion bien-pensante et fataliste. Il faut donc le libérer de la réification produite au fil du temps par l'écrit et le confier de nouveau à l'oralité rafraîchissante d'un conteur, à travers la musique. Le compositeur va de ce fait rejeter à la fois le caractère pré-donné du texte littéraire (en le déconstruisant phonétiquement), les systèmes musicaux pré-existants et l'usage conventionnel des sources sonores (instruments et voix), afin de réinventer le geste expressif, inventif, verbal aussi bien que musical, étouffé par le monde de la technique qui n'offre plus à la consommation qu'une matière pré-formée et des objets pré-fabriqués.

Comment donc ouvrir un accès totalement nouveau à une histoire qui ne devrait pas se borner à demeurer « touchante »? Tout au contraire, qui devrait convaincre d'agir !

La réponse de Lachenmann à cette question va être l'imbrication de l'objectivité et de la subjectivité dont parle Michel Leiris dans l'épigraphe de ce chapitre aussi bien que Walter Benjamin dans la citation précédente. C'est précisément cette coexistence paradoxale qui est propre au conte, un genre à la fois dépourvu de pathos dans la simplicité de son "dit» et profondément habité par la subjectivité du conteur qui transmet ainsi une expérience humaine incarnée. Ici, chez Lachenmann, les paroles prononcées par les voix sont celles du récit dans sa sobriété, mais elles sont profondément subjectivisées, non par une emphase affective qui ne serait finalement que représentation des affects ${ }^{20}$, mais par le canal sensitif de la musique dont on a vu à quel point elle transcrivait le vécu corporel et les perceptions multiples de l'enfant. La subjectivité manifeste ici sa présence non par l'analyse psychologique mais bien par l'évocation d'une expérience concrète.

19 Sur le plan du livret à proprement parler, les tropes sont juxtaposés au conte, sans préparation, comme froidement et en toute objectivité (le procédé de la citation et l'incroyable éloignement du discours révolté de Gudrun Ensslin et de l'expérience philosophique de Vinci renforcent le phénomène de distanciation), mais c'est leur teneur même qui trahit l'implication subjective du conteur (la trace de la main du potier sur le vase) et la mobilisation personnelle concrète qu'il espère du spectateur. La construction du livret par montage permet à Lachenmann à la fois de se prémunir du sentimentalisme inutile parce qu'inefficace qui pourrait naitre de la seule histoire touchante de la petite fille et de tempérer l'extra-territorialité froide des deux textes insérés. Il veut provoquer par leur juxtaposition inattendue un questionnement personnel sur la véritable implication de chacun dans les problèmes humains évoqués :

La lettre de Gudrun Ensslin que j'ai utilisée commence par ces mots : « Le criminel, le fou, le suicidé... », et avec ces trois mots, elle s'est définie elle-même. Il n'y a aucun doute que son action fût criminelle. Mais elle montre également qu'une telle chose peut nous rattraper tous. Ensslin venait du même milieu que moi, c'était une croyante, une idéaliste, très vive et en même temps aveuglée, qui a conçu très sérieusement le projet de changer la société. Elle a souffert de la froideur et de la chaleur factice de cette société, réagissant avec une violence qu'il faut assurément condamner. Mais quand on a un esprit un tant soit peu subtil, on doit sentir, audelà de la condamnation, le mécanisme social fatal qui est à l'œuvre et qui nous implique tous. Dans l'opéra, le texte d'Ensslin désigne l'un des trois topoi dont je me suis toujours préoccupé, trois états de souffrance en somme, que l'œuvre embrasse : l'enfant paria, qui meurt de froid, et qui dans son malheur, brûle les allumettes interdites; le rebelle qui se met lui-même à l'écart, qui désespère face à une société de plus en plus rigide et agit de manière violente, en sortant de la légalité; enfin celui qui cherche la connaissance, avec non moins de passion, mais qui sent ses 
limites devant une grotte qui lui inspire la peur autant qu'un désir d'y pénétrer. Ces

trois figures possibles de l'homme me semblent indissociables ${ }^{21}$. Lachenmann mise sur l'effet de déchirement qu'ils provoquent par leur montage dans la structure du récit, pour rappeler les déchirements de l'existence. Tout d'abord, un déchirement au sein de la mémoire individuelle : le conte d'Andersen rappelle à chacun son enfance avec ses émotions fortes; l'abandon à la peur qu'inspirait alors l'histoire de la petite fille était d'autant plus grand qu'il se passait dans les conditions protégées et affectives du moment délicieux où se déployait la narration. Mais dans l'œuvre de Lachenmann, cette mémoire sollicitée se trouve brutalement privée de la douceur du souvenir par l'intrusion de souffrances d'adultes; les deux textes insérés trouent le récit connu et le recouvrent de crissements d'une douloureuse actualité. D'autre part, un déchirement au sein de la mémoire collective: chaque tableau, qu'il provienne d'Andersen ou des propres choix de Lachenmann, souligne la solitude des êtres (la petite fille, Gudrun Ensslin, le philosophe Vinci) confrontés à l'hostilité de l'environnement social, à l'indifférence du cours du monde ou aux mystères de la Nature ; la mise en regard du total abandon de la petite et de la rage terroriste des années 70 semble projeter sur l'œuvre à la fois une haine du monde tel qu'il est et l'ombre de la culpabilité collective dont la génération de Lachenmann subit inexorablement encore la malédiction.

21 Ajoutons à cette "teneur en vérité $»^{22}$ du livret, celle - historique - de la technique expressionniste du montage, héritée d'un temps où les artistes luttaient contre l'écrasement de l'individu par la norme collective. Comme chez ses aînés expressionnistes, le montage auquel se livre Lachenmann dans la composition de son livret prévient toute tentation de mise en cohérence qui ne pourrait être, aujourd'hui encore, que mensongère. La permanence de ce procédé jusqu'à Lachenmann, malgré les mauvais procès intentés en Allemagne à l'expressionnisme après la tragédie du nazisme ${ }^{23}$, témoigne chez le compositeur d'un héritage courageusement assumé dans une décennie - les années 90 - où le pays médite une fois de plus douloureusement sur l'accumulation tragique des épisodes récents (la succession de deux totalitarismes et la difficile réunification dans le cadre d'une démocratie passionnément protégée) et sur sa nécessaire recomposition ${ }^{24}$.

deuleur intense, le vécu du déchirement, la réflexion politique, le sauvetage de l'individu contre le spectre des totalitarismes, la volonté de refondation sans reniement des blessures encore ouvertes de la mémoire..., tous ces thèmes traversent en filigrane l'opéra de Lachenmann, lui imprimant une force qui signale les grandes œuvres même si elles conservent leur caractère énigmatique. En cela, Lachenmann s'avère bien «le porte-venin", selon l'expression saisissante de Michel Leiris, de l'Allemagne contemporaine.

\section{La Petite Fille aux allumettes ou la libération de l'esprit}

Il semblera peut-être paradoxal de considérer une telle œuvre, si sombre et tourmentée, comme la promesse d'un éveil, d'une libération, et pourtant - on le perçoit à des signes multiples - Lachenmann l'a conçue ainsi. 

conte, quoique la fin de la petite fille revête chez lui des couleurs de rédemption et de salut chrétiens absentes - en tout cas en apparence - de la version lachenmannienne. La grand-mère (la Grande Mère) entrouvre à la petite la porte des cieux et du séjour des Bienheureux, abandonnant à leur indifférence à jamais impardonnable les bourgeois qui découvrent son cadavre au matin. Mais Lachenmann ne se situe pas tout à fait sur ce registre. En insérant ces tropes, il inscrit le conte dans l'ici-bas, dans une philosophie de l'existence qui cherche à faire jaillir du pêle-mêle des expériences humaines l'utopie d'une transformation du monde et puise la transcendance dans l'immanence même. C'est tout d'abord l'écrit nihiliste de Gudrun Ensslin qui offre des clés pour une interprétation dialectique du conte : par sa mort à la fois subie et assumée (souvent par le suicide), l'opprimé dénonce le crime hypocrite dont il est victime, se révolte et punit son bourreau (c'est en tout cas l'idée qu'en retient Lachenmann...). Vinci, de son côté, révèle, dans la même veine dialectique mais sur le versant humaniste cette fois, le double pouvoir du néant (l'obscurité de la caverne), à la fois générateur d'angoisse et déclencheur d'appétit de savoir. Les tropes semblent indiquer que la petite fille triomphe finalement de l'indifférence générale et que sa mort - par la sublime fin musicale, extatique - est en fin de compte initiation; Andersen y parvenait, lui, par la puissance de l'affectivité et le souvenir de la seule bonté rédemptrice que la petite ait jamais rencontrée, celle de sa grand-mère - avatar de l'Éternel féminin faustien -, plus fort que toute souffrance. Dans l'obscurité de la salle, le spectateur expérimente donc le face à face solitaire avec la petite, sans médiation autre que ses sens et quelques éclats énigmatiques de pensée fragmentée. L'interpellation de sa sensibilité s'effectue non plus par le phénomène de l'identification affective mais par une empathie puissante, d'ordre sensoriel, renforcée par « le rébus de la conscience fissurée ${ }^{25}$ que constitue le livret.

En second lieu, l'objectif de renouvellement de la perception par une musique inouïe, enracinée dans l'expérience sensorielle individuelle, signale, chez un compositeur qui se méfie des utopies (que ce soient Marx ou le Messie), l'existence d'une «utopie dialectique concrète, l'utopie vivante $»^{26}$, comme la décrivait Ernst Bloch, la conviction finalement optimiste qu'une transformation du présent est possible grâce à l'invention d'un monde-non-encore-advenu, restant à inventer. Il s'agit d'en revenir à l'être même, sans pathos, en silence, comme la petite fille qui, faisant fi de la prétendue richesse qu'elle a entre les mains, privilégie un imaginaire représentant ici-bas sa seule chance de bonheur.

Tout l'espoir de Lachenmann, déposé dans son œuvre aride, est là, dans la fabrication d'un sensible inédit, construit par des expériences sensorielles oubliées, qui mettent la conscience aux aguets et stimulent la pensée. En mobilisant chez son auditeur des facultés d'écoute nouvelles, libérées par la découverte de sonorités inconnues et pourtant profondément enracinées dans la vérité du corps et de l'affectivité, le pari de Lachenmann est de dépasser le simple stade du sentir pour accéder au stade ultérieur d'une perception qui fasse sens et devienne libération. Par ce processus, la transformation de l'homme et du monde est enclenchée et s'avère, dans l'esprit du compositeur, plus efficace que les dénonciations pratiquées par l'art engagé.

En concevant une musique inouïe pour laquelle les auditeurs n'ont aucune autre clé que leur propre perception, Lachenmann avive l'intensité d'un présent d'autant plus essentiel que toute mémoire consolatrice est devenue impossible depuis qu'elle s'est 
rompue dans les désastres de l'histoire individuelle et collective. Seule l'implication totale de l'individu dans le monde peut garantir contre l'absorption par une masse indifférente et permettre d'affronter l'inconnu en toute conscience. Extraire l'individu des ornières de la perception creusées dans un monde déshumanisé et l'amener à lâcher prise grâce à des facultés renouvelées de sentir et de méditer, tel est le projet de l'artiste. La mémoire est douloureuse, voire impossible; elle ne peut parvenir que par lambeaux. Soit! Par le livret, le montage de ces bribes servira de livre d'histoire, de livre d'histoires... Et si l'on recourt au conte traditionnellement ancré dans la nuit des temps, ce n'est pas par facilité ou tendance régressive, mais bien dans un but réflexif. Mémoire mutilée et perception renouvelée seront désormais les fondements d'un art actuel qui n'a qu'une ambition, celle d'armer l'homme contemporain contre toute menace de manipulation et de le tenir éveillé devant les désastres comme devant les merveilles du monde...

Un tel spectacle de la perception refuse donc l'endormissement par le plaisir de l'habitude ${ }^{27}$ et œuvre à l'éveil de l'esprit. Il dessine de nouvelles perspectives d'action politique, non plus motivée par une représentation idéale du collectif, mais par la réinvention d'un être qui s'est trouvé soit annihilé par la société de masse, soit mutilé sensoriellement par la technologie envahissante, soit perverti par l'égoïsme consumériste. Le partage d'un sensible libéré des manipulations pernicieuses de la société médiatique dévoilera l'inanité des bons sentiments qui n'ont jamais empêché les lecteurs de contes touchants de voir s'éteindre chaque jour des petites filles aux allumettes dans l'encoignure de leurs maisons.

Tous ceux qui ont enquêté sur le paupérisme se posent la question de savoir quand est atteinte la limite de la misère humaine, écrit Benjamin dans son livre sur Baudelaire. Que reste-t-il quand on n'a plus rien? L'unique expérience du monde! Dans La Petite Fille aux allumettes, Lachenmann semble montrer que lorsqu'il n'y a plus rien, pas même le logos pour combattre le chaos - soit que la parole ait été dévoyée (comme dans le nazisme, par exemple), soit qu'elle ait été réifiée par l'idéologie (comme chez Gudrun Ensslin), soit qu'elle ait été détruite par l'excès de souffrance (comme chez Celan, par exemple) -, demeure l'être-au-monde, extraordinaire réservoir de facultés perceptives capables de transformer la représentation que l'on a de l'univers. Et c'est précisément cette faculté de transformation que voudrait éveiller Lachenmann. Il s'agit pour lui de remettre l'expérience au centre de la vie afin de retrouver cette sensibilité qu'un monde réifié lui a enlevée. À l'Erlebnis, ensemble de sensations qui sous-tendent la contemplation de la petite fille devant la flamme éphémère, Lachenmann associe l' Erfahrung, méditation consciente sur le chemin de la vie. Cette refondation sur l'être originel, incarné par l'enfant souffrant, imprime à la pensée l'impulsion nécessaire pour accomplir le cheminement existentiel (Erfahren). L'élargissement sensoriel poursuivi par Lachenmann à travers son œuvre doit ainsi permettre à l'être de l'auditeur de se fortifier en dépassant la déconstruction du moi, métaphorisée par une forme artistique disloquée.

30 La petite Fille aux allumettes participe donc à sa manière d'une nouvelle poétique de l'opéra dominée depuis quelques décennies par la mise en question de la représentation classique et la focalisation sur la question de l'être qu'a si bien pointée Laurent Feneyrou ${ }^{28}$ :

Dans la tragédie, insiste Heidegger, à l'inverse d'Aristote dont jamais il ne cite la Poétique, il ne se passe rien. Antigone n'agit pas plus qu'elle n'interagit, car ce serait s'affairer dans l'étant et y oublier l'être ; sa seule " action ", poursuit Heidegger qui écrit, à dessein, le mot Handlung entre guillemets - est d'endurer le deinon [le 
terrible, mais aussi l'étrange [...]], c'est-à-dire d'assumer jusqu'à en mourir la vue de l'être en tant qu'il se destine à elle ». La tragédie ne produit donc rien, sinon la vérité d'une éloquence statique, née de l'effroi. [...]

Une telle ontologisation de la dramaturgie se retrouve dans l'opéra contemporain, plusieurs musiciens ayant étudié ces thèses sur la tragédie antique, et plus particulièrement sur l'Édipe roi et l'Antigone, à travers les commentaires de Hölderlin et de Heidegger. Le renoncement à l'intrigue, aux rôles déterminés, la dislocation des personnages, indifféremment confiés à des solistes, à un ensemble ou à un chœur, voire à des instruments, et les scissions des livrets dans l'écriture et le chant, l'avènement d'une scène sans histoire ou d'une tragédie sans action, autorisent à élargir les dialogues entre Poésie et Pensée. Et la scène, désertée [...], le parcours des infinis chemins sans station, sans but [...], créent un lieu de l'errance, $\mathrm{du}$ malheur, dépourvu de toute fable. L'écoute y pourfend l'essence de la représentation [...].

La banalisation contemporaine du visuel et du sonore, la tendance, accrue par le numérique, à la virtualisation de l'expérience expliquent que, par réaction, bon nombre d'œuvres actuelles cherchent à attirer l'attention sur un sensible débarrassé tant des excès de la cérébralité pure que de l'émotion frelatée. L'écoute exigeante est alors souvent privilégiée comme résistance à l'intrusion de l'image et à la passivité de l'audition. On le constate notamment dans la "tragédie de l'écoute», sous-titre de l'opéra Prometeo de Nono ou dans «l'action invisible» du Lohengrin de Sciarrino. La problématisation du livret devient alors un puissant adjuvant à la musique pour soutenir cette quête exploratoire. Souvent contesté dans sa configuration verbale, déconstruit afin de le prémunir des interprétations routinières, donné à percevoir plus qu'à comprendre, il se fond à la musique pour faire émerger des modes nouveaux de perception du réel qui se trouve par là réinventé.

Néanmoins, dans le cas qui nous intéresse ici, ne faisons pas comme si l'œuvre avait déjà été intégrée, tout naturellement, par la culture contemporaine! Ne faisons pas comme si la description et l'interprétation suffisaient à l'apprivoiser et à nous l'approprier! Ne laissons pas croire que l'écoute de cette musique difficile se fait confortablement ! Ne donnons pas à penser que le livret se laisse approcher sans peine, lui qui, paradoxalement, sauve de la destruction linguistique imposée au conte deux écrits qui se trouvent de ce fait légitimés à parts égales, celui d'une terroriste et celui d'un humaniste ! Ce serait afficher une superbe déplacée, méprisant les réelles difficultés d'accès sur lesquelles buteront encore certainement, et à juste titre, bon nombre de spectateurs! Il ne sert à rien de nier les nombreux questionnements qu'engendre cette œuvre troublante et ardue. Osons plutôt avouer avec humilité une certaine perplexité, tout en reconnaissant que le débat suscité contribue à distinguer cette œuvre complexe des objets de consommation culturelle avec lesquels elle ne risque heureusement pas d'être confondue, gardant par là sa capacité d'interpellation!

Ce Märchen, de par son titre que nous connaissons si bien, nous a en effet rassemblés dans le temple de l'art qu'est l'Opéra, et pourtant - à notre grande surprise ! - son sens s'est dérobé et nous a laissés désemparés. C'est notamment que le Conteur a disparu, avec ce qui faisait de lui un rassembleur d'humanité, la langue! La langue, ce bien commun qui nous a toujours permis de tendre la main au passé et d'échanger des expériences avec nos semblables, s'est émiettée, dissoute, nous abandonnant à l'effroi de la solitude originelle. N'est-ce pas cette catastrophe - la disparition du logos, ce bien commun qui assurait le partage d'expériences - que met en scène Lachenmann dans sa Petite Fille aux allumettes? N'est-ce pas ce désarroi symbolique, hérité de l'après Seconde 
Guerre mondiale, qui est pour la nième fois rappelé ici ? Tantôt falsifiée, tantôt effacée, tantôt " assassinée " ${ }^{29}$, tantôt dénoncée comme complice de la barbarie, la langue est mise en pièces, comme dans beaucoup d'œuvres musicales contemporaines. Lorsqu'elle demeure entière, comme dans les tropes, c'est sous une apparence totalement réifiée, enserrée par une musique qui en souligne l'inanité. Pourtant, n'est-ce pas au prix de cette déconstruction métaphorique du sujet à travers l'émiettement de la langue que l'esprit parvient à se libérer ? Comme l'écrit Martin Kaltenecker citant à son tour le compositeur, «Le lieu musical de Lachenmann, le basho ${ }^{30}$, est en même temps le champ d'une dialectique et d'une déconstruction : de Dieu, de la Musique ou du Sujet, « ce moi qui est un mirage funeste que l'on doit prendre à revers, au nom d'un secret, d'une réalité, d'une présence qui ne se révèlent pas autrement, et dont pourtant provient toute force ${ }^{31} »$.

Ainsi une promesse de bonheur affleure dans la fin transfigurée : la musique retrouve, par l'intermédiaire de la sonorité " chantée » (et non plus bruitée), peu familière à nos oreilles occidentales, de l'orgue à bouche et des longues tenues harmoniques, une fluidité et une continuité qui font oublier les soubresauts pathétiques du langage désarticulé entendu jusque là. Il semble que l'être se déprenne ici de sa souffrance et parvienne au vide lumineux, à un présent limpide, là où le combat contre la langue, contre tout système figé, contre tout dogme, devient inutile. Cette pacification ultime laisse espérer qu'un état heureux peut advenir et puisque le temps historique semble avoir été détruit par cet art écorché qui récuse l'héritage du passé, peut-être qu'un ailleurs spatial, « le lieu du rien $\|^{32}$, métaphorisé par l'instrument japonais, se laisse-t-il enfin entrevoir? Et ce lieu est le lieu même de l'esprit. La distance parcourue entre l'obsession nihiliste de la mort - subie, donnée, contemplée - qui culmine sans doute dans la fin cataclysmique des Soldats (1965) de Zimmermann ${ }^{33}$, et la fin sublimement apaisée de La Petite Fille aux allumettes serait-elle un premier symptôme de guérison ? En tout cas, chez Lachenmann, le vertige du néant qui habite l'œuvre semble se dissoudre in extremis dans la découverte ultime de la transcendance, une quête que le compositeur reconnaît ${ }^{34}$ et qui motive sans doute son attachement ineffaçable au conte d'Andersen.

À travers cette grande œuvre, l'art allemand entreverrait-il - et la culture allemande avec lui ${ }^{35}$ - la possibilité d'un apaisement de ses tourments ? Se pourrait-il que, par le renouveau de la perception rêvé par Lachenmann, il soit bientôt en mesure de transformer la désespérante noirceur de sa tonalité psychique pour trouver la libération salvatrice?

\section{NOTES}

1. Helmut Lachenmann, cité par Martin Kaltenecker in: Avec Helmut Lachenmann, Paris, Van Dieren Editeur, 2001, p. 238.

2. Helmut Lachenmann, considéré comme l'un des compositeurs contemporains les plus marquants, est né à Stuttgart en 1935. 
3. L'œuvre fut créée, sur commande de l'Opéra, à Hambourg, en 1997. Il en existe un enregistrement audio: Das Mädchen mit den Schwefelhölzern, Tokyo-Fassung, 2000, SWR Sinfonieorchester, Sylvain Cambreling, ECM New Series 1858/59, 4761283.

4. "J'ai d'ailleurs un autre projet, et un jour, sans tenir compte des commandes en cours et de tout ce qui vient toujours retarder les choses, je vais le mettre à exécution : composer une œuvre scénique, appelez cela opéra, même si c'est inexact [...]. Je partirai de l'histoire touchante du conte d'Andersen sur la petite fille aux allumettes, mais cela sera tout sauf touchant. Je considérerai ce projet comme une sorte d'aboutissement de mon travail jusqu'ici, où ma musique devra s'éployer de manière aussi populaire (!) que possible, sans concessions bien sûr, pour exprimer quelque chose. » H. Lachenmann, lettre à Rudolf Lück du 8 juillet 1975, citée par Martin Kaltenecker in : Avec Helmut Lachenmann, Paris, Van Dieren Editeur, 2001, p. 225- 226.

5. On peut en prendre la mesure dans la correspondance du compositeur concernant la genèse de l'œuvre publiée par Thomas Schäfer dans le livre programme de l'Opéra de Hambourg en 1997.

6. "Lorsque je conçois une musique de manière à ce qu'elle ne soit pas là a priori, mais se construise progressivement, de façon structurée, à travers les paramètres qui la composent, le son de la voix humaine est un élément extrêmement dérangeant - il représente un état que je veux justement arriver à créer. » Lachenmann (1997), cité par Kaltenecker, op. cit., p. 233.

7. Ce trope ne concerne pas seulement le livret puisque Lachenmann insère dans l'opéra une œuvre musicale, Zwei Gefühle. Musik mit Leonardo, composée en 1992, pendant la difficile gestation de l'œuvre scénique.

8. Par exemple, en liant la dernière lettre d'un mot à la première syllabe du mot suivant.

9. Lachenmann, cité par Kaltenecker, op. cit., p. 235-236.

10. Lettre de Lachenmann à Kaltenecker, 16 mai 2001, in Kaltenecker, op. cit., p. 237.

11. « Le criminel, le fou, le suicidé, ils incarnent cette contradiction, ils en crèvent. Leur mort rend évidentes l'absence d'issue et l'impuissance de l'homme dans le système : soit tu te détruis toi-même soit tu en détruis d'autres - tu es soit mort, soit égoïste. Dans leur mort n'apparaît pas seulement la perfection du système. Ils ne sont pas assez criminels, pas assez fous, pas assez meurtriers. Et cela veut dire : une mort plus rapide causée par le système. Alors leur mort montre en même temps la négation du système : leur criminalité, leur folie, leur mort seront l'expression de la révolte d'un sujet détruit contre sa destruction, qui n'est plus un objet, mais un être humain. » Gudrun Ensslin, 1973, cité et traduit par Kaltenecker, op. cit., p. 243.

12. "La tempête ne mugit pas avec un plus formidable bruit de tonnerre quand le vent du nord cinglant l'agite et fait déferler les vagues entre Charybde et Scylla. De même le Stromboli et l'Etna ne sont pas plus violents lorsque le feu de soufre ouvre la grande montagne pour projeter dans les airs les pierres et la terre jaillissantes et propulsées avec les flammes. De même les cavernes incandescentes de Mongibello quand elles expulsent les éléments qui résistaient à leur furie débridée...

Mais j'erre alentour, poussé par mon avidité brûlante de percevoir le grand désordre des formes étranges et variées que la nature intelligente a produites. Je me faufile entre les rochers ombreux jusqu'à parvenir à l'entrée d'une grande caverne devant laquelle je demeure un moment dans un sentiment d'incertitude. J'avance le dos voûté, la main gauche posée sur le genou, la droite protégeant mes yeux. Et alors que je me penche pour scruter l'intérieur de la caverne et y distinguer quelque chose, j'en suis empêché par la profonde obscurité qui y règne. Mais après avoir persisté un certain temps, s'éveillèrent en moi deux sentiments : la crainte et le désir. La crainte de l'obscurité menaçante de la caverne, mais aussi le désir de voir de mes propres yeux ce qu'il pouvait s'y trouver de merveilleux ». Léonard de Vinci, Codex Arundel (Traduit par nos soins avec l'aide de Claire Lecointre que je remercie).

13. «Le montage est précisément la clé de toute bizarrerie, c'est la description du pêle-mêle de la réalité vécue avec des sphères effondrées et des césures [...], un désordre qui cherche sa forme chez Protée, dans le pêle-mêle de la nature en fermentation, et non plus chez Prométhée, dans le 
sujet en fermentation expressive. Aujourd'hui, tout cela n'est que le rébus de la conscience fissurée, avec une totalité qui trouve son tout dans des lambeaux, dans les ricochets d'une réalité vécue sans apprêts. » Ernst Bloch, Héritage de ce temps, Paris, Payot, 1978 (en allemand, Erbschaft der Zeit, Frankfurt am Main, Suhrkamp Verlag, 1935 puis 1962), p. 208.

14. Pour plus de détails sur l'œuvre, on lira avec profit l'excellent livre de Martin Kaltenecker, déjà cité maintes fois, p. 225 à 270.

15. Der Gesang der Jünglinge de Stockhausen, composé en 1956 au studio de Cologne est l'une des premières œuvres électroniques. Elle s'appuie sur Le Livre de Daniel et évoque la louange adressée à Dieu par les enfants jetés dans la fournaise. Dans une évocation terrifiante, des voix d'enfants parviennent par bribes comme si elles perçaient un univers crépitant rendu par les sons électroacoustiques.

16. Michel Leiris, L'Afrique fantôme, Paris, Gallimard, 1934, p. 210.

17. «[...] l'art de conter est en train de se perdre. Il est de plus en plus rare de rencontrer des gens qui sachent raconter une histoire. [...] C'est comme si nous avions été privés d'une faculté qui nous semblait inaliénable, la plus assurée d'entre toutes: la faculté d'échanger des expériences. » Walter Benjamin, «Le Conteur. Réflexions sur l'œuvre de Nicolas Leskov », in: CEuvres III, Paris, Gallimard, 2000, p. 115.

18. « Le Conteur... », ibid., p. 127.

19. Ibid., p. 133.

20. On ne peut s'empêcher ici de citer le célèbre texte de Roland Barthes « Le Grain de la voix », qui se montre si étonnamment proche de ce qui vient d'être décrit, in : L'Obvie et l'obtus, Paris, Seuil, 1982, p. 241-242 : «Le " grain » de la voix n'est pas - ou n'est pas seulement - son timbre : la signifiance qu'il ouvre ne peut précisément mieux se définir que par la friction même de la musique et d'autre chose, qui est la langue (et pas du tout le message). [...] Comparons deux morts chantées - fort célèbres toutes deux, celle de Boris et celle de Mélisande. Quelles qu'aient été les intentions de Moussorgski, la mort de Boris est expressive, ou, si l'on préfère, hystérique ; elle est surchargée de contenus affectifs, historiques; toutes les exécutions de cette mort ne peuvent être que dramatiques : c'est le triomphe du phéno-texte, l'étouffement de la signifiance sous le signifié d'âme. Mélisande au contraire, ne meurt que prosodiquement. [...] Entre les deux, un creux bienfaisant, qui faisait le plein de Boris : le pathos, c'est-à-dire selon Aristote (pourquoi pas ?), la passion telle que les hommes la parlent, l'imaginent, l'idée reçue de la mort, la mort endoxale. Mélisande meurt sans bruit : rien ne vient troubler le signifiant, et donc rien n'oblige à la redondance ; il y a production d'une langue-musique dont la fonction est d'empêcher le chanteur d'être expressif. "

21. Lachenmann, cité par Kaltenecker, op. cit., p. 244.

22. Wahrheitsgehalt, un concept-clé de la Théorie esthétique d'Adorno.

23. On pourra lire à ce sujet mon article: "L'Expressionnisme à la question ", in : Destruction, création, rythme: l'expressionnisme, une esthétique du conflit, édité par Georges Bloess, Paris, L'Harmattan, 2007 (à paraître).

24. Lire à ce sujet l'excellent livre de Jacques-Pierre Gougeon, Allemagne: une puissance en mutation, Paris, Gallimard, 2006.

25. Ernst Bloch, Héritage de ce temps, op. cit., p. 208.

26. Ernst Bloch, Ibid., "Sur l'histoire originale du Troisième Reich », p. 137.

27. Le refus de l'habitude est, pour Lachenmann, la définition même de la beauté.

28. Laurent Feneyrou, «En deçà d'Aristote. Dramaturgies hölderliniennes dans le drame lyrique de la fin du $\mathrm{xx}^{\mathrm{e}}$ siècle ", in : Composer un opéra aujourd'hui, sous la direction de Béatrice RamautChevassus, Publications de l'Université de Saint-Etienne, 2003.

29. On visionnera avec grand intérêt l'impressionnant documentaire de Nurith Aviv, D'une langue à l'autre 55 ', 2004. qui témoigne du rapport difficile des hommes traumatisés par l'histoire du $\mathrm{xx}^{\mathrm{e}}$ siècle avec la langue. 
30. La notion de basho, «Logique du lieu », est développée par le grand philosophe japonais de l'expérience pure qu'est Nishida. Celui-ci, imprégné de phénoménologie occidentale autant que de philosophie zen, a profondément marqué Lachenmann par sa pensée du vide.

31. Lachenmann, cité par Kaltenecker, op. cit., p. 319.

32. Titre du dernier ouvrage de Nishida, Logique du lieu du rien, 1945.

33. Compositeur allemand d'avant-garde, contemporain exact de Lachenmann.

34. "Le dépassement de l'œuvre par une "situation musicale », qui marque donc d'une certaine manière la mort de la musique, a été mis en rapport par le compositeur en 1996 avec l'adage nietzschéen de la mort de Dieu. Dans un discours prononcé à Donaueschingen, Lachenmann remarque qu'une telle proclamation, paradoxalement, n'était possible qu'à un esprit passionnément religieux, à la recherche de Dieu et en fin de compte profondément croyant. », Kaltenecker, op. cit., p. 318.

35. C'est en tout cas la thèse de Jacques-Pierre Gougeon dans Allemagne : une puissance en mutation, op. cit.

\section{RÉSUMÉS}

Le célèbre conte d'Andersen constitue le socle du livret du compositeur Helmut Lachenmann, pour son œuvre scénique de 1997. Mais comment les propriétés du conte peuvent-elles s'allier à la dramaturgie de l'opéra contemporain, avec sa tendance à disloquer les textes, à expurger la scène de toute action, à dissoudre les personnages dans le jeu instrumental et à ontologiser le genre à tel point que l'écoute pure devient l'enjeu principal de l'œuvre au détriment de toute représentation? C'est pourtant ce que va tenter de réaliser Lachenmann, en faisant de sa «musique concrète instrumentale» le véritable narrateur, le passeur d'expérience. La caractéristique du conte était en effet d'allier la sobriété pudique du récit à l'interprétation subjective du conteur qui faisait ainsi parvenir jusqu'au présent l'expérience des temps passés. Lachenmann ne confie pas la narration à une voix soliste, mais va faire percevoir au spectateur le ressenti même de la petite fille transie, et ce, grâce à une musique qui s'emploie à retranscrire les sensations corporelles de l'enfant. Le compositeur se propose ainsi d'élargir l'expérience sensorielle de l'auditeur afin de renouveler ses capacités perceptives et de transformer en conséquence un rapport au monde anesthésié par le "retrait de l'expérience» dont parle Benjamin. Ce «spectacle de la perception» devient alors un véritable projet politique, non pas art engagé sur le mode de la dénonciation, mais tentative de transformation de l'humain dont le salut dépend de son accès au spirituel.

Das berühmte Märchen Andersens bildet die Grundlage des Librettos des Komponisten Helmut Lachenmann für sein Bühnenstück von 1997. Aber wie können die Charakteristika des Märchens mit einer zeitgenössischen Operndramaturgie in Einklang gebracht werden, mit ihrer Tendenz, die Texte auseinanderzureißen, jegliche Handlung auf der Bühne zu streichen, die Figuren im Spiel der Instrumente aufzulösen und die Gattung so sehr zu ontologisieren, daß es im Werk nun mehr um das reine Hören geht zu Lasten der Darstellung? Doch gerade das versucht Lachenmann umzusetzen, indem er aus seiner «konkreten Instrumentalmusik» den echten Erzähler, den Überbringer von Erfahrungen macht. Das Charakteristische des Märchens war, die diskrete Schmucklosigkeit der Erzählung mit der subjektiven Interpretation des Erzählers zu verbinden, wodurch die Erfahrungen vergangener Zeiten übermittelt wurden. Lachenmann überträgt die 
Erzählung nicht auf eine Solostimme, sondern er läßt dank einer Musik, die für den Ausdruck der körperlichen Wahrnehmungen des Kindes eingesetzt wird, den Zuschauer die Empfindungen des erstarrten Mädchens nachfühlen. Der Komponist beabsichtigt auf diese Weise, die sinnlichen Erfahrungen des Hörers zu erweitern, damit er seine perspektivischen Fähigkeiten erneuert und dementsprechend den Bezug zur betäubten Welt durch «die Entauratisierung», von der Benjamin spricht,verändert.

Dieses «Wahrnehmungsstück» wird daraufhin zu einem echten politischen Projekt, es ist nicht engagierte Kunst über die Art und Weise des Anprangerns, sondern der Versuch, den Menschen zu ändern, dessen Heil vom Zugang zum Geistigen abhängig ist.

INDEX

Mots-clés : musique concrète instrumentale

oeuvrecitee La petite fille aux allumettes

\section{AUTEURS}

JOËLLE CAULLIER

Université Charles-de-Gaulle - Lille 3 